\title{
Euler's sum of powers conjecture
}

Euler's conjecture is a disproved conjecture in mathematics related to Fermat's last theorem. It was proposed by Leonhard Euler in 1769. ${ }^{[1]}$ It states that for all integers $n$ and $k$ greater than 1 , if the sum of $n$ $k$ th powers of positive integers is itself a $k$ th power, then $n$ is greater than or equal to $k$ :

$$
a_{1}^{k}+a_{2}^{k}+\ldots+a_{n}^{k}=b^{k} \Rightarrow n \geq k
$$

The conjecture represents an attempt to generalize Fermat's last theorem, which is the special case $n=2$ : if $a_{1}^{k}+a_{2}^{k}=b^{k}$, then $2 \geq k$.

Although the conjecture holds for the case $k=3$ (which follows from Fermat's last theorem for the third powers), it was disproved for $k=4, k=5, k=7$, and $k=8$. It is unknown whether the conjecture fails or holds for any value $k \geq 6$.

\section{Contents}

Background

Counterexamples

Generalizations

$k=3$

$k=4$

$k=5$

$k=7$

$k=8$

See also

References

External links

\section{Background}

Euler was aware of the equality $59^{4}+158^{4}=133^{4}+134^{4}$ involving sums of four fourth powers; this however is not a counterexample because no term is isolated on one side of the equation. He also provided a complete solution to the four cubes problem as in Plato's number $3^{3}+4^{3}+5^{3}=6^{3}$ or the taxicab number 1729. ${ }^{[2][3]}$ The general solution of the equation

$$
x_{1}^{3}+x_{2}^{3}=x_{3}^{3}+x_{4}^{3}
$$

is

$$
\begin{array}{cl}
x_{1}=1-(a-3 b)\left(a^{2}+3 b^{2}\right), & x_{2}=(a+3 b)\left(a^{2}+3 b^{2}\right)-1 \\
x_{3}=(a+3 b)-\left(a^{2}+3 b^{2}\right)^{2}, & x_{4}=\left(a^{2}+3 b^{2}\right)^{2}-(a-3 b)
\end{array}
$$


where $a$ and $b$ are any integers.

\section{Counterexamples}

Euler's conjecture was disproven by L. J. Lander and T. R. Parkin in 1966 when, through a direct computer search on a CDC 6600, they found a counterexample for $k=5 .{ }^{[4]}$ This was published in a paper comprising just two sentences. ${ }^{[4]}$ A total of three primitive (that is, in which the summands do not all have a common factor) counterexamples are known:

$$
\begin{aligned}
& \left.27^{5}+84^{5}+110^{5}+133^{5}=144^{5} \text { (Lander \& Parkin, 1966) }\right)^{[4]} \\
& (-220)^{5}+5027^{5}+6237^{5}+14068^{5}=14132^{5} \text { (Scher \& Seidl, 1996), and } \\
& 55^{5}+3183^{5}+28969^{5}+85282^{5}=85359^{5} \text { (Mufassaroh \& Setiawan, 2020) }
\end{aligned}
$$

In 1986, Noam Elkies found a method to construct an infinite series of counterexamples for the $k=4$ case. ${ }^{[6]}$ His smallest counterexample was

$$
2682440^{4}+15365639^{4}+18796760^{4}=20615673^{4} .
$$

A particular case of Elkies' solutions can be reduced to the identity[7][8]

$$
\left(85 v^{2}+484 v-313\right)^{4}+\left(68 v^{2}-586 v+10\right)^{4}+(2 u)^{4}=\left(357 v^{2}-204 v+363\right)^{4}
$$

where

$$
u^{2}=22030+28849 v-56158 v^{2}+36941 v^{3}-31790 v^{4}
$$

This is an elliptic curve with a rational point at $v_{1}=-\frac{31}{467}$. From this initial rational point, one can compute an infinite collection of others. Substituting $v_{1}$ into the identity and removing common factors gives the numerical example cited above.

In 1988, Roger Frye found the smallest possible counterexample

$$
95800^{4}+217519^{4}+414560^{4}=422481^{4}
$$

for $k=4$ by a direct computer search using techniques suggested by Elkies. This solution is the only one with values of the variables below $1,000,000 .^{[9]}$

\section{Generalizations}

In 1967, L. J. Lander, T. R. Parkin, and John Selfridge conjectured ${ }^{[10]}$ that if

$$
\sum_{i=1}^{n} a_{i}^{k}=\sum_{j=1}^{m} b_{j}^{k},
$$

where $a_{i} \neq b_{j}$ are positive integers for all $1 \leq i \leq n$ and $1 \leq j \leq m$, then $m+n \geq k$. In the special case $m=1$, the conjecture states that if

$$
\sum_{i=1}^{n} a_{i}^{k}=b^{k}
$$


(under the conditions given above) then $n \geq k-1$.

The special case may be described as the problem of giving a partition of a perfect power into few like powers. For $k=4,5,7,8$ and $n=k$ or $k-1$, there are many known solutions. Some of these are listed below. As of 2002, there are no solutions for $k=6$ whose final term is $\leq 730000$ [11] $^{[1}$

$k=3$

$$
\left.3^{3}+4^{3}+5^{3}=6^{3} \text { (Plato's number } 216\right)
$$

This is the case $a=1, b=0$ of Srinivasa Ramanujan's formula

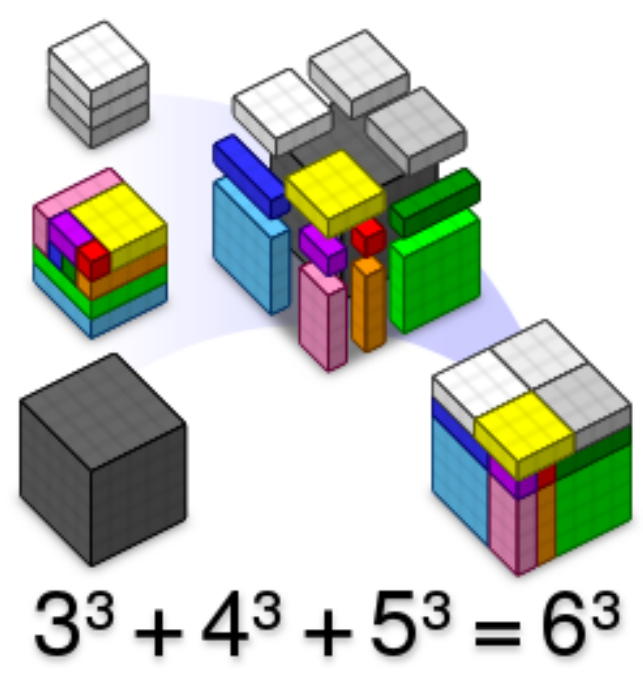

One interpretation of Plato's number, $3^{3}+4^{3}+5^{3}=6^{3}$

$$
\begin{aligned}
& \left(3 a^{2}+5 a b-5 b^{2}\right)^{3}+\left(4 a^{2}-4 a b+6 b^{2}\right)^{3}+\left(5 a^{2}-5 a b-3 b^{2}\right)^{3}=\left(6 a^{2}-4 a b+4 b^{2}\right)^{3} \\
& \text { [12] }
\end{aligned}
$$

A cube as the sum of three cubes can also be parameterized as

$$
a^{3}\left(a^{3}+b^{3}\right)^{3}=b^{3}\left(a^{3}+b^{3}\right)^{3}+a^{3}\left(a^{3}-2 b^{3}\right)^{3}+b^{3}\left(2 a^{3}-b^{3}\right)^{3}
$$

or as

$$
a^{3}\left(a^{3}+2 b^{3}\right)^{3}=a^{3}\left(a^{3}-b^{3}\right)^{3}+b^{3}\left(a^{3}-b^{3}\right)^{3}+b^{3}\left(2 a^{3}+b^{3}\right)^{3} \cdot[12]
$$

The number $2100000^{3}$ can be expressed as the sum of three cubes in nine different ways. ${ }^{[12]}$

$$
\boldsymbol{k}=\mathbf{4}
$$

$$
\begin{aligned}
& 95800^{4}+217519^{4}+414560^{4}=422481^{4}(\mathrm{R} . \text { Frye, } 1988)^{[6]} \\
& 30^{4}+120^{4}+272^{4}+315^{4}=353^{4}(\mathrm{R} . \text { Norrie, } 1911)^{[10]}
\end{aligned}
$$

This is the smallest solution to the problem by R. Norrie.

$$
\boldsymbol{k}=\mathbf{5}
$$$$
27^{5}+84^{5}+110^{5}+133^{5}=144^{5}(\text { Lander \& Parkin, 1966) })^{[13]}
$$$$
19^{5}+43^{5}+46^{5}+47^{5}+67^{5}=72^{5} \text { (Lander, Parkin, Selfridge, smallest, 1967) }{ }^{[10]}
$$

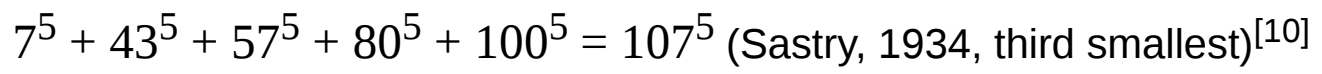

$$
k=7
$$


$127^{7}+258^{7}+266^{7}+413^{7}+430^{7}+439^{7}+525^{7}=568^{7}$ (Mufassaroh \& Setiawan, 2020) $)^{[14]}$

$k=8$

$90^{8}+223^{8}+478^{8}+524^{8}+748^{8}+1088^{8}+1190^{8}+1324^{8}=1409^{8}$ (Mufassaroh \& Setiawan, 2020) ${ }^{[15]}$

\section{See also}

- Jacobi-Madden equation

- Prouhet-Tarry-Escott problem

- Beal's conjecture

- Pythagorean quadruple

- Generalized taxicab number

- Sums of powers, a list of related conjectures and theorems

\section{References}

1. Mufassaroh, Arij Zulfi; Setiawan, Adib Rifqi (2020). "Incorrect Mathematical Conjecture" (http s://thesiscommons.org/3pnxc/). Thesis Commons. Open Science Framework (OSF). 19 April:

1. doi:10.31237/osf.io/3pnxc (https://doi.org/10.31237\%2Fosf.io\%2F3pnxc).

2. Dunham, William, ed. (2007). The Genius of Euler: Reflections on His Life and Work (https://bo oks.google.com/books?id=M4-zUnrSxNoC\&pg=PA220). The MAA. p. 220. ISBN 978-0-88385558-4.

3. Titus, III, Piezas (2005). "Euler's Extended Conjecture" (http://www.oocities.org/titus_piezas/Eq ualsums.htm).

4. Lander, L. J.; Parkin, T. R. (1966). "Counterexample to Euler's conjecture on sums of like powers". Bull. Amer. Math. Soc. 72 (6): 1079. doi:10.1090/S0002-9904-1966-11654-3 (https://d oi.org/10.1090\%2FS0002-9904-1966-11654-3).

5. Mufassaroh, Arij Zulfi; Setiawan, Adib Rifqi (2020). "Counterexample to Euler's Conjecture for Fifth Power" (https://thesiscommons.org/ybh6k/download). Thesis Commons. 19 April: 1. doi:10.31237/osf.io/ybh6k (https://doi.org/10.31237\%2Fosf.io\%2Fybh6k).

6. Elkies, Noam (1988). "On $A^{4}+B^{4}+C^{4}=D^{4 " ~(h t t p: / / w w w . a m s . o r g / j o u r n a l s / m c o m / 1988-51-18 ~}$ 4/S0025-5718-1988-0930224-9/S0025-5718-1988-0930224-9.pdf) (PDF). Mathematics of Computation. 51 (184): 825-835. doi:10.1090/S0025-5718-1988-0930224-9 (https://doi.org/10. 1090\%2FS0025-5718-1988-0930224-9). JSTOR 2008781 (https://www.jstor.org/stable/200878 1). MR 0930224 (https://www.ams.org/mathscinet-getitem?mr=0930224).

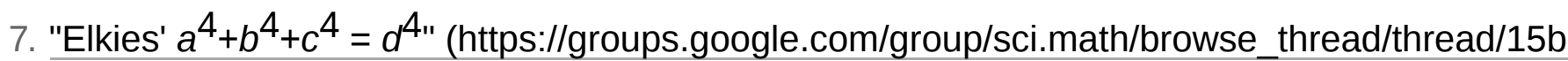
eef75eaddcb1b?hl=en\#).

8. "Sums of Three Fourth Powers" (https://sites.google.com/site/tpiezas/014).

9. Frye, Roger E. (1988), "Finding $95800^{4}+217519^{4}+414560^{4}=422481^{4}$ on the Connection Machine", Proceedings of Supercomputing 88, Vol.II: Science and Applications, pp. 106-116, doi:10.1109/SUPERC.1988.74138 (https://doi.org/10.1109\%2FSUPERC.1988.74138)

10. Lander, L. J.; Parkin, T. R.; Selfridge, J. L. (1967). "A Survey of Equal Sums of Like Powers". Mathematics of Computation. 21 (99): 446-459. doi:10.1090/S0025-5718-1967-0222008-0 (htt ps://doi.org/10.1090\%2FS0025-5718-1967-0222008-0). JSTOR 2003249 (https://www.jstor.or g/stable/2003249). 
11. Giovanni Resta and Jean-Charles Meyrignac (2002). The Smallest Solutions to the Diophantine Equation $a^{6}+b^{6}+c^{6}+d^{6}+e^{6}=x^{6}+y^{6}$ (http://www.ams.org/journals/mcom/2 003-72-242/S0025-5718-02-01445-X/S0025-5718-02-01445-X.pdf), Mathematics of Computation, v. 72, p. 1054 (See further work section).

12. Math world : Diophantine Equation--3rd Powers (http://mathworld.wolfram.com/DiophantineEq uation3rdPowers.html)

13. Burkard Polster (March 24, 2018). "Euler's and Fermat's last theorems, the Simpsons and CDC6600" (https://www.youtube.com/watch?v=AO-W5aEJ3Wg) (video). Retrieved 2018-03-24.

14. Mufassaroh, Arij Zulfi; Setiawan, Adib Rifqi (2020). "Counterexample to Euler's Conjecture for Seventh Power" (https://thesiscommons.org/9yg3j/download). Thesis Commons. 19 April: 1. doi:10.31237/osf.io/9yg3j (https://doi.org/10.31237\%2Fosf.io\%2F9yg3j).

15. Mufassaroh, Arij Zulfi; Setiawan, Adib Rifqi (2020). "Counterexample to Euler's Conjecture for Eighth Power" (https://thesiscommons.org/m27pc/download). Thesis Commons. 19 April: 1. doi:10.31237/osf.io/m27pc (https://doi.org/10.31237\%2Fosf.io\%2Fm27pc).

\section{External links}

- Tito Piezas III, A Collection of Algebraic Identities (https://sites.google.com/site/tpiezas/Home/)

- Jaroslaw Wroblewski, Equal Sums of Like Powers (http://www.math.uni.wroc.pl/ jwr/eslp/)

- Ed Pegg Jr., Math Games, Power Sums (https://web.archive.org/web/20080410224256/http:// www.maa.org/editorial/mathgames/mathgames_11_13_06.html)

- James Waldby, A Table of Fifth Powers equal to a Fifth Power (2009) (http://pat7.com/jp/s51510007-t)

- R. Gerbicz, J.-C. Meyrignac, U. Beckert, All solutions of the Diophantine equation $a^{6}+b^{6}=c^{6}$ $+d^{6}+e^{6}+f^{6}+g^{6}$ for $a, b, c, d, e, f, g<250000$ found with a distributed Boinc project (https://arxi v.org/abs/1108.0462)

- EulerNet: Computing Minimal Equal Sums Of Like Powers (http://euler.free.fr/)

- Weisstein, Eric W. "Euler's Sum of Powers Conjecture" (https://mathworld.wolfram.com/Eulers SumofPowersConjecture.html). MathWorld.

- Weisstein, Eric W. "Euler Quartic Conjecture" (https://mathworld.wolfram.com/EulerQuarticCon jecture.html). MathWorld.

- Weisstein, Eric W. "Diophantine Equation--4th Powers" (https://mathworld.wolfram.com/Diopha ntineEquation4thPowers.html). MathWorld.

- Euler's Conjecture (https://web.archive.org/web/20071105172444/http://library.thinkquest.org/2 8049/Euler\%27s\%20conjecture.html) at library.thinkquest.org

- A simple explanation of Euler's Conjecture (http://www.mathsisgoodforyou.com/conjecturesthe orems/eulerconjecture.htm) at Maths Is Good For You!

Retrieved from "https://en.wikipedia.org/w/index.php?title=Euler\%27s_sum_of_powers_conjecture\&oldid=951936461"

This page was last edited on 19 April 2020, at 18:08 (UTC).

Text is available under the Creative Commons Attribution-ShareAlike License; additional terms may apply. By using this site, you agree to the Terms of Use and Privacy Policy. Wikipedia ${ }^{\circledR}$ is a registered trademark of the Wikimedia

Foundation, Inc., a non-profit organization. 